\title{
LIVING RING OPENING METATHESIS POLYMERIZATION OF MESOGENIC NORBORNENE DERIVATIVES
}

\author{
Coleen Pugh
}

Department of Chemistry, The University of Michigan, Ann Arbor, MI 481091055, USA

\begin{abstract}
A new class of SCLCPs was prepared by polymerizing mesogenic norbornene derivatives using $\mathrm{Mo}\left(\mathrm{CHCMe}_{2} \mathrm{R}\right)\left(\mathrm{N}-2,6-\mathrm{C}_{6} \mathrm{H}_{3}-i-\mathrm{Pr}_{2}\right)(\mathrm{O}-t-\mathrm{Bu})_{2}(\mathrm{R}=$ $\mathrm{CH}_{3}$ or $\mathrm{Ph}$ ). Monomers based on norbornene ring systems were chosen because the rings are highly strained and therefore yield irreversible polymerizations. The Mo-alkylidene initiators were chosen because they initiate norbornene derivatives relatively fast and quantitatively, and provide stable chain ends which have low reactivity to both the internal double bonds of the polymer backbone and the functional groups present in the monomers. The apparent absence of termination and transfer reactions in ROMP results in polymerizations which appear to be living, and the fast initiation and irreversible chain growth leads to polymers with narrow molecular weight distributions in which the degree of polymerization is controlled by the ratio of monomer to initiator used. The resulting well-defined polymers were used to determine the most basic structure-property relationships of this new class of SCLCPs. The thermotropic behavior of both terminally attached and laterally attached SCLCPs based on polynorbornene backbones becomes independent of molecular weight at approximately 25 repeat units. In addition, polydispersity was found to have no effect on the breadth of nematic phase transitions in the terminally attached polymers, with the transition temperature determined simply by the number average degree of polymerization.
\end{abstract}

\section{INTRODUCTION}

Although numerous side chain liquid crystalline polymers (SCLCPs) have been prepared since their discovery in the early 1970's, elucidation of their elementary synthetic and structural principles has only recently begun $(1,2)$. This is because most SCLCPs have been prepared by methods in which it is not possible to control only one structural variable at a time; i.e. methods such as hydrosilation of mesogenic olefins with polysiloxanes and radical polymerization of acrylates and methacrylates. However, living polymerizations can now be used to determine the effect of a chosen structural feature, while other variables such as molecular weight and polydispersity remain constant. Therefore, any new class of SCLCPs will ideally also be synthesized by a living technique in which the molecular weight dependence of the thermal transitions can be reliably established, and in which the influence of polydispersity can be minimized. This paper reviews the synthesis and characterization of a new class of SCLCPs prepared by living ring opening metathesis polymerization (ROMP) of mesogenic norbornene derivatives. In addition, the mechanism of ROMP and its elementary reactions are reviewed in order to demonstrate what reagents and reaction conditions are required to obtain well-defined SCLCPs using this polymerization method.

\section{RING OPENING METATHESIS POLYMERIZATION}

Ring opening metathesis polymerization $(3,4)$ of cycloolefins is a chain polymerization, and therefore involves the four elementary reactions shown in Scheme I. In addition to initiation 


\section{Scheme I}

Initiation

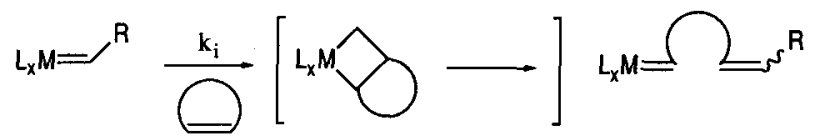

Propagation

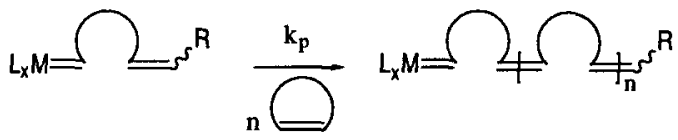

Transfer
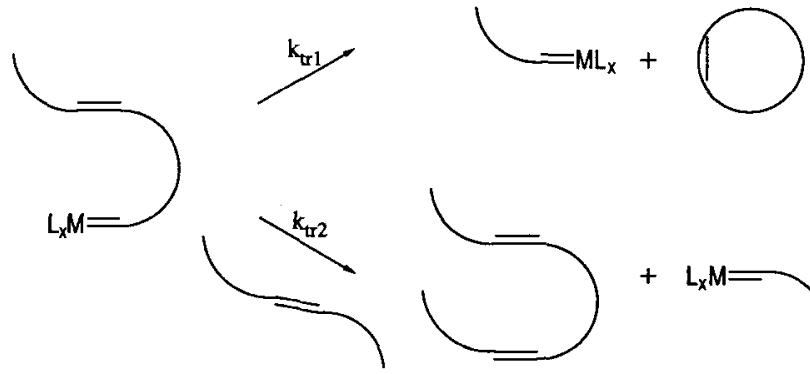

$+L_{x} M=$

Termination
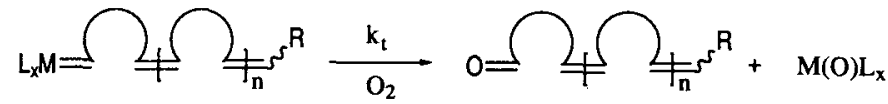

and propagation, chain polymerizations may also undergo transfer and termination reactions; the latter two reactions must be eliminated to obtain a living system. Classic initiating systems for ROMP involve a transition metal such as $\mathrm{WCl}_{6}, \mathrm{WOCl}_{4}$ or $\mathrm{MoCl}_{5}$, combined with an alkyl metal such as $n$-BuLi or a Lewis acid such as $\mathrm{AlEtCl}_{2}, \mathrm{SnMe}_{4}$ or $\mathrm{SnPh}_{4}$. In this case, a low concentration of a metallaolefin is generated in situ, which reacts with the cycloolefinic monomer with a rate constant $\mathbf{k}_{i}$ by a [2+2] cycloaddition to form a metallocyclobutane intermediate, which then ring opens to regenerate a metallaolefin at the terminus of a linear olefin. The overall ring opening of the cyclic monomer therefore occurs by cleavage of the double bond. Propagation proceeds in the same way with a rate constant $k_{p}$ by successive monomer insertions into the metal-carbon double bond at the growing chain ends. Although the growing metallaolefin is generally the detectable chain end when well-defined ROMP initiators are used, the detectable propagating species using bis $\left(\eta^{5}\right.$ cyclopentadienyl)titanacyclobutane (5) and tris(phenoxide)tantallacyclobutane (6) derivatives 
are the metallocyclobutanes. However, both the metal-alkylidene and metallocyclobutane propagating chain ends, as well as their interconversion, were observed by low temperature NMR in the $\mathrm{W}(\mathrm{CH}-t-\mathrm{Bu})\left(\mathrm{OCH}_{2}-t-\mathrm{Bu}_{2} \mathrm{Br}_{2}: \mathrm{GaBr}_{3}\right.$ initiated polymerization of norbornene (7) and the $\mathrm{Mo}(\mathrm{CHR})\left(\mathrm{N}-2,6-\mathrm{C}_{6} \mathrm{H}_{3}-i-\mathrm{Pr}_{2}\right)\left(\mathrm{OR}^{\prime}\right)_{2}$ initiated polymerizations of some substituted norbornadienes (8).

Each repeat unit of the polymers prepared by ring opening metathesis polymerization of cycloolefins contains a double bond which may participate in secondary metathesis reactions. Therefore, the most troublesome chain transfer reaction is chain transfer to polymer. Intramolecular chain transfer to polymer results in macrocycle formation with elimination of a shorter linear chain endcapped with the metallaolefin (or metallocyclobutane). Intermolecular chain transfer to polymer results in randomization of the chain lengths and broadening of the molecular weight distribution, presumably to the most probable distribution $\left(M_{w} / M_{n}=2\right)$.

Molecular oxygen and sometimes the carbonyl groups of aldehydes, ketones, esters and amides terminate the active chain ends. Benzaldehyde, pivaldehyde and acetone are often used as endcapping agents in living polymerizations; benzophenone is less efficient (9). As shown in Scheme I, reaction of the growing chain with molecular oxygen generates a metal oxide and an aldehyde terminated polymer. The resulting aldehyde chain end can then react with the active end of another polymer chain to generate a second molecule of the metal oxide and a polymer of higher molecular weight (10). This results in the double molecular weight fraction observed in polymerizations using well-defined initiators. Since the rate of reaction with molecular oxygen is slower than the rate of propagation, it occurs at the end of the polymerization (10).

\section{LIVING RING OPENING METATHESIS POLYMERIZATION}

The single requirement for a polymerization to be living is that all chain breaking reactions such as termination and transfer are absent (11-13); ideally $k_{t r}=k_{t}=0$. As shown in Figure 1 for a polymerization which is first order in monomer, a change in the number of active species due to termination is detected by a nonlinear dependence of monomer conversion as a function of time. However, termination will not be detected if it is slow on the time scale of the polymerization $\left(k_{t}<<k_{p}\right)$. That is, monomer conversion (eq. 1) will be complete if $k_{p} / k_{t} \geq$
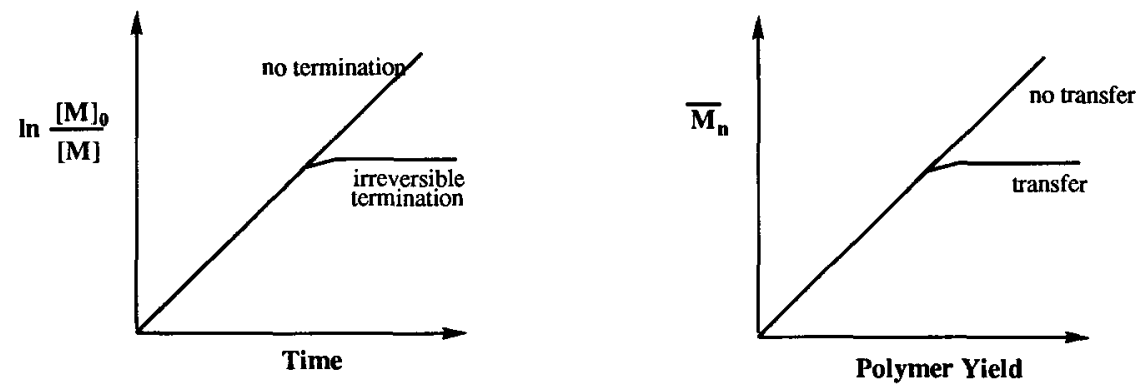

Figure 1. The effect of termination and transfer reactions on monomer conversion and polymer molecular weight. 
$10 \mathrm{x}[\mathrm{M}]_{0} /[\mathrm{I}]_{0} \sim 10 \mathrm{xDP}_{\mathrm{n}}(13)$. Termination is therefore less important at lower ratios of monomer to initiator.

$$
\ln \left([\mathrm{M}]_{0} /[\mathrm{M}]_{\infty}\right)=[\mathrm{I}]_{0} \mathrm{k}_{\mathrm{p}} / \mathrm{k}_{\mathrm{t}}
$$

The classic ROMP initiators usually generate low concentrations of unstable metallaolefin complexes which decompose during the polymerization (14). Therefore, in addition to eliminating molecular oxygen from the system, it is also necessary to use an initiating system which generates stable chain ends in order to prevent termination. Polymers with stable chain ends are generated when stable, well-defined, initiators containing either a metallocyclobutane or a metallaolefin are used $(5-9,14,15)$. Most of the initiators which produce well-defined polymers are high oxidation state complexes of titanium, tungsten or molybdenum. However, the active chain ends and initiators containing metallocyclobutanes rapidly decompose once monomer is consumed at the temperatures $\left(50-75^{\circ} \mathrm{C}\right)$ required to open the metallocyclobutane in chain growth $(5,6)$. In contrast, the coordinatively unsaturated molybdenum and tungsten alkylidene initiators developed by Schrock et al. $(14,16-18)$ are stable for weeks in an inert atmosphere. As shown in Scheme II, the metal center is stabilized by bulky tertiary alkoxide ligands and an imido ligand with bulky isopropyl substituents at the ortho positions.

In addition to the absence of termination, living conditions require that transfer reactions are also absent. Transfer reactions do not affect the number of active species, and are therefore not detected by following the monomer conversion if the rate of reinitiation is comparable to that of propagation. Instead, transfer affects the number of chains present in the system and is therefore detected by following the polymer molecular weight as a function of monomer conversion or polymer yield (Figure 1). However, a linear dependence of molecular weight vs. conversion is often used erroneously to demonstrate that a polymerization is living $(12,13)$. That is, termination does not affect the number of chains in the system, and therefore does not affect $\mathrm{M}_{\mathbf{n}}$ or $\mathrm{DP}_{\mathbf{n}}$ if initiation is fast and monomer conversion goes to completion; i.e. if all of the chains are not terminated. In addition, even transfer is difficult to detect if $k_{p} / k_{t r} \geq 10 x[M]_{0} /[]_{0}$ (13), and polymers with close to the

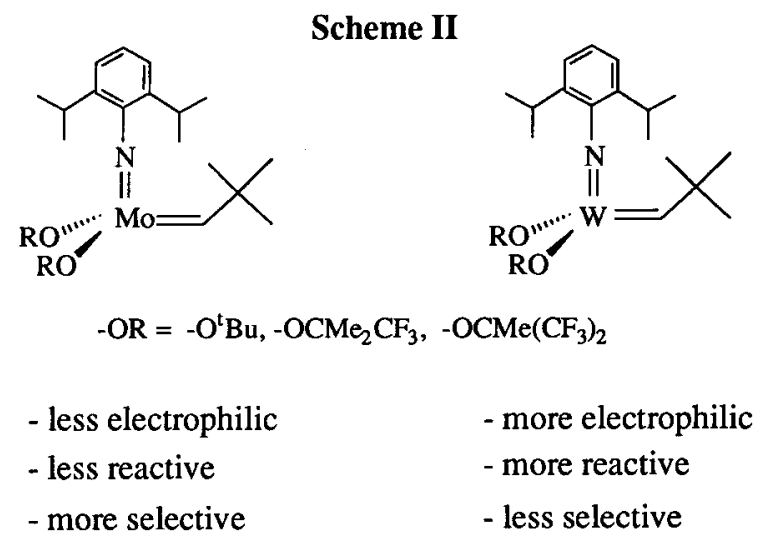


expected molecular weight will still be obtained under these conditions if initiation is fast and quantitative. Due to the ease of hiding transfer and termination by working at low ratios of monomer to initiator, Matyjaszewski has proposed calling a polymerization living only if $k_{p} / k_{t}$ and $k_{p} / k_{t r}$ are greater than $10^{4}$, or at least to rank the livingness based on the ratios of the rate constants of propagation and transfer or termination (13).

Neither the rate constants of transfer and termination, nor their respective ratios with the rate constant of propagation have been determined for "living" ring opening metathesis polymerization. However, polymers with $\mathrm{DP}_{\mathbf{n}}$ up to 500 are routinely obtained using Schrock's metal-alkylidene initiators (16-18), which is quite adequate for the synthesis of SCLCPs. In addition, the ability to prepare well-defined polymers with $\mathrm{DP}_{\mathrm{n}}=500$ requires that $k_{p} / k_{t} \geq 5 \times 10^{3}$ and/or $k_{p} / k_{t r} \geq 5 \times 10^{3}$, which is close to the requirements of a living polymerization proposed by Matyjaszewski.

As discussed in the previous section, chain transfer to polymer by secondary metathesis reactions are the most obvious transfer reactions which must be eliminated to obtain welldefined polymers by ROMP. These transfer reactions can be minimized by working with derivatives of bicyclic monomers such as bicyclo[2.2.1] hept-2-ene (norbornene) which yield polymers which are resistant to secondary metathesis due to steric hindrance (3). Secondary metathesis is also prevented by using initiators which do not metathesize internal olefins. That is, if the metallaolefin or metallocyclobutane complex is reactive enough to metathesize an internal olefin, it is too reactive to obtain a living polymerization. For example, W(CH-t$\mathrm{Bu})\left(\mathrm{N}-2,6-\mathrm{C}_{6} \mathrm{H}_{3}-i-\mathrm{Pr}_{2}\right)\left[\mathrm{OCMe}_{2}\left(\mathrm{CF}_{3}\right)_{2}\right]_{2}$ will metathesize cis-2-pentene at over 1000 turnovers per minute in toluene at $25^{\circ} \mathrm{C}$ but will not produce a living polynorbornene, whereas $\mathrm{W}(\mathrm{CH}-\mathrm{t}$ $\mathrm{Bu})\left(\mathrm{N}-2,6-\mathrm{C}_{6} \mathrm{H}_{3}-i-\mathrm{Pr}_{2}\right)(\mathrm{O}-t-\mathrm{Bu})_{2}$ will produce a living polynorbornene although it metathesizes cis-2-pentene at only $\sim 2$ turnovers per hour at $25^{\circ} \mathrm{C}$ (14).

The reactivity and selectivity of Schrock's metal-alkylidene initiators are summarized in Scheme II. In general, the metal center of the molybdenum alkylidenes are less electrophilic than the corresponding tungsten alkylidenes, and are therefore less reactive and more selective. However, the electrophilicity of the metal center and its reactivity is increased by more electron withdrawing ligands, such as $-\mathrm{OCMe}\left(\mathrm{CF}_{3}\right)_{2}$ vs. $t$-butoxide. $\mathrm{Mo}(\mathrm{CH}-t-\mathrm{Bu})(\mathrm{N}-2,6-$ $\left.\mathrm{C}_{6} \mathrm{H}_{3}-i-\mathrm{Pr}_{2}\right)(\mathrm{O}-t-\mathrm{Bu})_{2}$ is the least reactive complex shown, and therefore the most selective initiator for ROMP. It tolerates a variety of non-protonic functional groups in norbornene and norbornadiene monomers $(19,20)$ and in the endcapping agent (21), especially if THF is used as the solvent. Such groups include acetate, cyano, ethers, esters, $\mathrm{N}$-substituted imides and trifluoromethyl groups. However, the more reactive $\mathrm{Mo}(\mathrm{CH}-\mathrm{t}-\mathrm{Bu})\left(\mathrm{N}-2,6-\mathrm{C}_{6} \mathrm{H}_{3}-i-\mathrm{Pr}_{2}\right)-$ $\left(\mathrm{OCMe}_{2} \mathrm{CF}_{3}\right)_{2}$ is sometimes required to polymerize functionalized 7-oxanorbornadienes (8).

Although the absence of termination and transfer are the only requirements for a polymerization to be living, initiation must also be fast and quantitative (ideally $\mathrm{k}_{\mathrm{i}}=\infty$ ) in order to control the molecular weight by the ratio of monomer to initiator (eq. 2). If initiation is

$$
\mathrm{DP}_{\mathrm{n}}=\Delta[\mathrm{M}] /[\mathrm{I}]_{0}=\left([\mathrm{M}]_{0}-[\mathrm{M}]_{0}\right) /[\mathrm{I}]_{0}
$$

slow, the number average molecular weight is initially too high, but becomes equal to the theoretical value once both initiator and monomer are consumed. In practice. the number of 
growing chains will equal the amount of initiator used if the rate constant of initiation is of the same order of magnitude as that of propagation $\left(k_{i} \geq k_{p}\right)(22)$. This is demonstrated by the ring opening metathesis polymerizations of norbornene and norbornadiene derivatives using $\mathrm{Mo}(\mathrm{CH}-t-\mathrm{Bu})\left(\mathrm{N}-2,6-\mathrm{C}_{6} \mathrm{H}_{3}-i-\mathrm{Pr}_{2}\right)(\mathrm{O}-t-\mathrm{Bu})_{2}$, in which $\mathrm{k}_{\mathrm{p}} / \mathrm{k}_{\mathrm{i}}=12$ (23) and 0.7-30 (19c), respectively. The rate constant of initiation is less than that of propagation apparently because
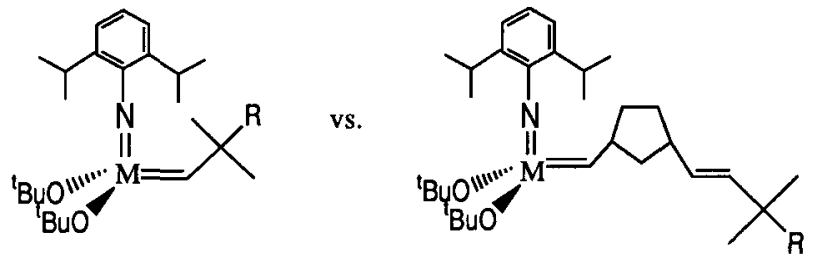

the neopentylidene ligand with a tertiary carbon $\beta$ to the metal is more sterically demanding than the insertion products, which contain secondary carbons $\beta$ to the metal.

Two additional requirements must be met in order to also obtain polymers with narrow molecular weight distributions approaching the Poisson distribution (eq. 3): all growing chain

$$
\mathrm{DP}_{\mathrm{w}} / \mathrm{DP}_{\mathrm{n}} \cong 1+1 / \mathrm{DP}_{\mathrm{n}}
$$

ends must have equal reactivity (either $k_{p}{ }^{x}=k_{p} y=k_{p}{ }^{z}$ etc., or the active sites must exchange rapidly), and the growth must be irreversible $\left(k_{p}>k_{d}\right)$. A ring opening polymerization will be irreversible if the monomer is highly strained. There are therefore two ring systems that may lead to well-defined polymers using ROMP: cyclobutene derivatives, and derivatives of the bicyclo[2.2.1]hept-2-ene ring system, including norbornenes, norbornadienes, 7 oxanorbornenes and 7-oxanorbornadienes. The cyclobutene systems have the disadvantage that the double bonds in the resulting polymers are highly susceptible to secondary metathesis reactions. However, well-defined polymers of cyclobutene were recently obtained by adding a Lewis base to a $\mathrm{W}(\mathrm{CH}-t-\mathrm{Bu})\left(\mathrm{N}-2,6-\mathrm{C}_{6} \mathrm{H}_{3}-i-\mathrm{Pr}_{2}\right)(\mathrm{O}-t-\mathrm{Bu})_{2}$ initiated polymerization, thereby decreasing the reactivity of the initiator and growing chain ends, and also increasing the rate of initiation relative to that of propagation (24). So far, only mesogenic norbornene derivatives initiated by $\mathrm{Mo}\left(\mathrm{CHCMe}_{2} \mathrm{R}\right)\left(\mathrm{N}-2,6-\mathrm{C}_{6} \mathrm{H}_{3}-i-\mathrm{Pr}_{2}\right)(\mathrm{O}-\mathrm{t}-\mathrm{Bu})_{2}\left(\mathrm{R}=\mathrm{CH}_{3}\right.$ or $\left.\mathrm{Ph}\right)$ have been used to prepare well-defined SCLCPs. Thus, well-defined SCLCPs can be prepared by polymerizing highly strained norbornene derivatives in an oxygen and moisture-free environment using a molybdenum imido alkylidene initiator. In this case, the strained monomer leads to irreversible chain growth and polymers with hindered double bonds along the backbone, whereas the chosen initiator gives relatively fast initiation and stable chain ends which do not react with internal olefins and many functional groups.

The initiator(s) are prepared as outlined in Scheme III in an inert atmosphere $(17,18)$. Any pentane used throughout the synthesis must be washed with acid to remove contaminating olefins, and it and all other solvents and reagents must be dry. The first high yield synthesis of the molybdenum imido alkylidene complexes started with $\mathrm{MoO}_{2}$, which was chlorinated at high temperature $\left(160^{\circ} \mathrm{C}\right.$ ) in a gas flow reactor (17). The resulting $\mathrm{MoO}_{2} \mathrm{Cl}_{2}$ was then converted quantitatively to $\mathrm{Mo}\left(\mathrm{N}-2,6-\mathrm{C}_{6} \mathrm{H}_{3}-i-\mathrm{Pr}_{2}\right)_{2} \mathrm{Cl}_{2}$ (dme) by reacting it with 2 equiv of 2,6- 


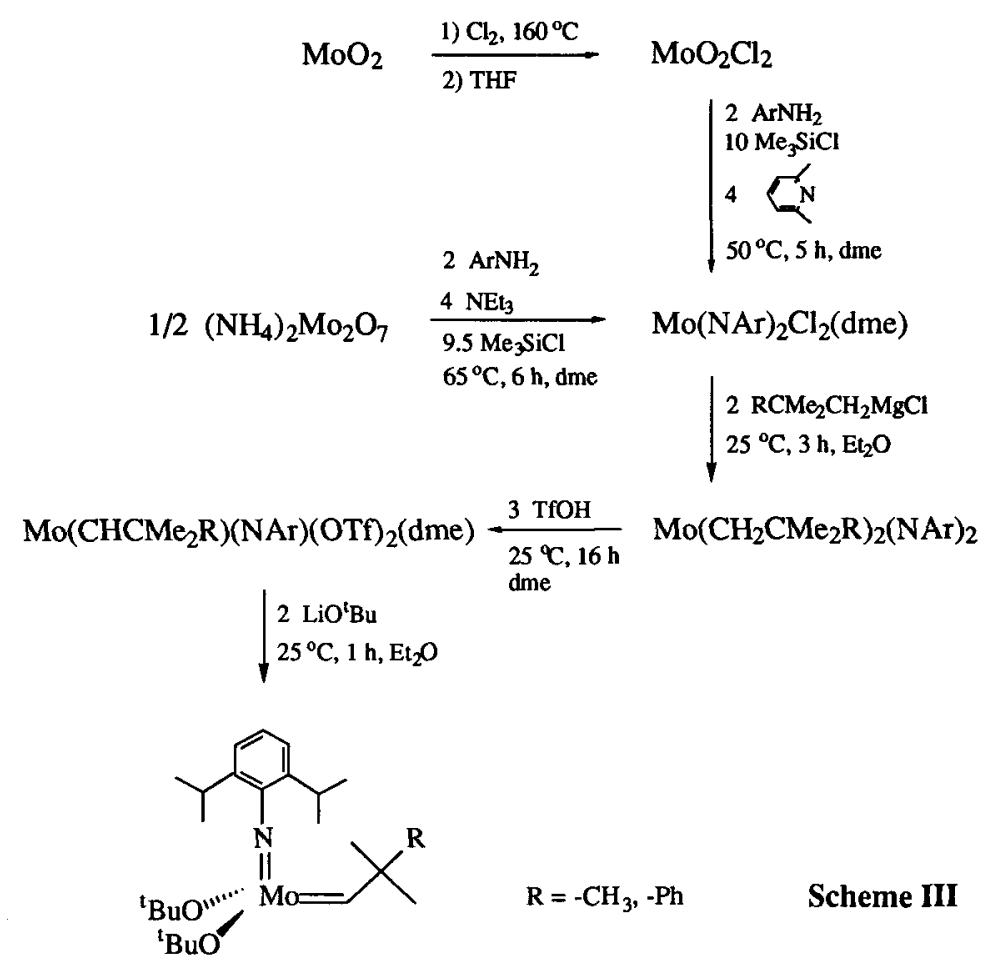

diisopropylaniline in dimethoxyethane in the presence of 2,6-lutidine as base and excess trimethylchlorosilane. Although the exact role of trimethylchlorosilane is not known, it appears to convert oxo ligands to chlorides with formation of hexamethyldisiloxane (17). The overall role of trimethylchlorosilane and 2,6-lutidine is therefore to consume the $\mathrm{HCl}$ and water generated by the reaction.

$\mathrm{Mo}\left(\mathrm{N}-2,6-\mathrm{C}_{6} \mathrm{H}_{3}-i-\mathrm{Pr}_{2}\right)_{2} \mathrm{Cl}_{2}$ (dme) can also be prepared on a larger scale directly from inexpensive ammonium dimolybdate and 2,6-diisopropylaniline in dimethoxyethane, using triethylamine as base and excess trimethylchlorosilane (18). The chloride ligands are then displaced by either neopentyl magnesium chloride $\left(\mathrm{R}=-\mathrm{CH}_{3}\right)$ or neophyl magnesium chloride $(\mathrm{R}=-\mathrm{Ph})(17)$; the neophyl route has the advantages that the Grignard reagent is easier and less expensive to prepare, and the resulting complexes with neophyl ligands are easier to crystallize. Reaction of $\mathrm{Mo}\left(\mathrm{CH}_{2} \mathrm{CMe}_{2} \mathrm{R}\right)_{2}\left(\mathrm{~N}-2,6-\mathrm{C}_{6} \mathrm{H}_{3}-i-\mathrm{Pr}_{2}\right)_{2}$ with 3 equiv of triflic acid produces $\mathrm{Mo}\left(\mathrm{CHCMe}_{2} \mathrm{R}\right)\left(\mathrm{N}-2,6-\mathrm{C}_{6} \mathrm{H}_{3}-i-\mathrm{Pr}_{2}\right)(\mathrm{OTf})_{2}(\mathrm{dme})$ via $\alpha$-hydrogen abstraction of the presumed intermediate $\mathrm{Mo}\left(\mathrm{CH}_{2} \mathrm{CMe} 2 \mathrm{R}\right)\left(\mathrm{N}-2,6-\mathrm{C}_{6} \mathrm{H}_{3}-i-\mathrm{Pr}_{2}\right)_{2}(\mathrm{OTf})_{2}$ (14). This product is the key intermediate to metathesis catalysts and initiators of varying reactivities. $\mathrm{Mo}\left(\mathrm{CHCMe}_{2} \mathrm{R}\right)\left(\mathrm{N}-2,6-\mathrm{C}_{6} \mathrm{H}_{3}-i-\mathrm{Pr}_{2}\right)(\mathrm{O}-t-\mathrm{Bu})_{2}$ is prepared by displacing the two triflate ligands with lithium $t$-butoxide, and recrystallizing the resulting ROMP initiator from pentane. 


\section{TERMINALLY ATTACHED SCLCPS}

Norbornene monomers containing terminally attached $p$-methoxybiphenyl (25-27) and $p$ cyanobiphenyl (27) mesogens were polymerized using $\mathrm{Mo}(\mathrm{CH}-t-\mathrm{Bu})\left(\mathrm{N}-2,6-\mathrm{C}_{6} \mathrm{H}_{3}-i-\mathrm{Pr}_{2}\right)(\mathrm{O}-t$ $\mathrm{Bu})_{2}$ in order to determine the effect of molecular weight, polydispersity and flexible spacer length on the thermotropic behavior of SCLCPs with polynorbornene backbones. The number

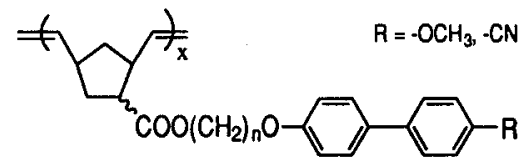

average degree of polymerization as calculated by GPC relative to polystyrene standards varied from 6 to 342, with polydispersities of 1.05-1.27. The higher polydispersities were usually the result of a minor amount of a double molecular weight fraction in addition to the expected molecular weight, due to insufficient degassing of molecular oxygen.

The polynorbornenes containing $p$-methoxybiphenyl mesogens with spacers of $n=2-8$ exhibit enantiotropic nematic mesophases in addition to the glass transition (25). The lower molecular weight samples with $n=9-10$ and the entire molecular weight range of samples with $n=11-12$ also undergo side chain crystallization (26). However, the mesophase of the $n=11-12$ samples appears to be smectic rather than nematic. In most cases, the monomers contained a mixture of 90:10 endo:exo substituted norbornenes. Varying this ratio from 90:10 to 75:25 endo:exo had no effect on the glass and nematic-isotropic transition temperatures of the $n=6$ sample. However, increasing the exo content of the monomers with $n=9-12$ may depress side chain crystallization in the resulting polymers; this is being checked.

Surprisingly, all of the polymers containing $p$-cyanobiphenyl mesogens exhibit only a glass transition and an enantiotropic nematic mesophase at spacer lengths of $n=4-12$ (27). That is, neither a smectic mesophase which is common for SCLCPs with p-cyanobiphenyl mesogens, nor side chain crystallization which is common for longer spacer lengths (1), are observed. The thermotropic behavior of all of the SCLCPs containing either $p$-cyano- or $p$ methoxybiphenyl mesogens attached to the polynorbornene backbone by an ester linkage become independent of molecular weight at DP=30-50 (25-27). The nearly monodisperse samples of the polymers with $p$-methoxybiphenyl mesogens and $n=6,7$ were blended to obtain polydisperse and multimodal samples $(n=6, P D I=2.50 ; n=7, P D I=4.78)$. The polydispersity had no effect on the peak width of isotropization nor on the temperature of the transitions (25). That is, the polydisperse samples underwent isotropization over a narrow temperature range as did the more monodisperse samples, and the temperatures of the glass and isotropization transitions corresponded to those of monodisperse samples with the same number average degree of polymerization.

Norbornenes with the $p$-methoxybiphenyl mesogen attached to the norbornene ring through an ether linkage have also been polymerized using $\mathrm{Mo}(\mathrm{CH}-t-\mathrm{Bu})\left(\mathrm{N}-2,6-\mathrm{C}_{6} \mathrm{H}_{3}-i\right.$ $\left.\operatorname{Pr}_{2}\right)(\mathrm{O}-t-\mathrm{Bu})_{2}(27)$. Although all of the resulting polymers were reported to be crystalline or semi-crystalline (27), the polymer with $\mathrm{n}=5$ was also prepared using classic ROMP initiators 


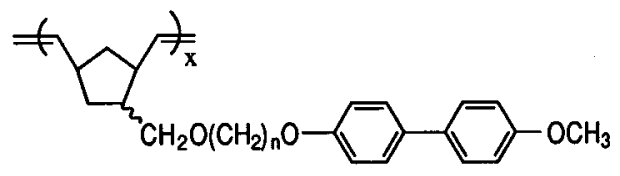

\begin{tabular}{|c|c|c|c|c|c|c|}
\hline \multirow[b]{2}{*}{$\underline{n}$} & \multicolumn{3}{|c|}{ GPC } & \multicolumn{2}{|c|}{ Phase Transitions $\left({ }^{\circ} \mathrm{C}\right)$} & \multirow[b]{2}{*}{ Ref. } \\
\hline & DPn & $\mathrm{Mn}$ & PDI & heating & cooling & \\
\hline 4 & 118 & 44700 & 1.15 & g $54 \mathrm{k} 108 \mathrm{i}$ & i 88 k 42 & 27 \\
\hline $\begin{array}{l}5 \\
5\end{array}$ & $\begin{array}{r}202 \\
69\end{array}$ & $\begin{array}{l}79500 \\
27000\end{array}$ & $\begin{array}{l}1.14 \\
2.40\end{array}$ & $\begin{array}{l}\text { k } 92 \mathrm{i} \\
\text { s } 88 \mathrm{i}\end{array}$ & $\begin{array}{l}\text { i } 73 \mathrm{k} \\
\text { i } 72 \mathrm{~s}\end{array}$ & $\begin{array}{l}27 \\
28\end{array}$ \\
\hline 6 & 122 & 49400 & 1.12 & k $94 \mathrm{i}$ & i $78 \mathrm{k}$ & 27 \\
\hline
\end{tabular}

and was reported to exhibit a highly ordered smectic mesophase (28). However, the transition temperatures of the two reports are nearly identical, and the latter paper demonstrated by polarized optical microscopy that a liquid crystalline phase formed.

\section{LATERALLY ATTACHED SCLCPS}

Most SCLCPs with laterally attached mesogens exhibit only nematic mesophases, including those based on poly(methylsiloxane)s, polyacrylates, polymethacrylates and polychloroacrylates. Small angle neutron scattering experiments demonstrated that the mesogens in a nematic mesophase "jacket" the backbone, which is forced into an extended helical conformation due to the large difference in size of the mesogens compared to the repeat unit of the backbone (30). Since these intriguing SCLCPs were prepared by living polymerizations, there was no information on the molecular weight dependence of the thermotropic behavior of monodisperse samples. The following polynorbornenes were

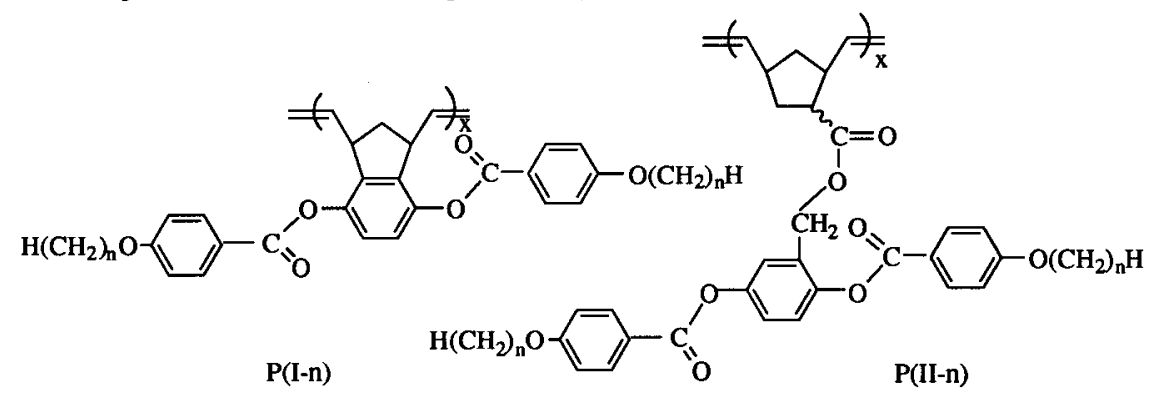

prepared by ROMP of the corresponding norbornene monomers using $\mathrm{Mo}\left(\mathrm{CHCMe}_{2} \mathrm{Ph}\right)(\mathrm{N}-$ 2,6- $\left.\mathrm{C}_{6} \mathrm{H}_{3}-i-\mathrm{Pr} 2\right)(\mathrm{O}-t-\mathrm{Bu})_{2}$ in order to determine the effect of molecular weight and the effect of the length of the n-alkoxy chain on the thermotropic behavior of laterally attached SCLCPs (31). The bis[(4'-n-alkoxybenzoyl)oxy]phenyl mesogens were used in order to determine if ROMP can be used to polymerize norbornene derivatives containing phenylbenzoate groups in a living or controlled manner. This would be advantageous considering the ease of preparing phenylbenzoate mesogens with a number of structural variations, yet the inability of polymerizing monomers containing these mesogens by a living anionic or group transfer polymerizations. Finally, polymers $\mathrm{P}(\mathrm{I}-\mathrm{n})$ with the mesogen fused to the backbone were 
prepared to test the concept that direct attachment of the mesogen should favor mesophase formation (32), which is in contrast to terminally attached SCLCPs which usually require a spacer.

The number average degree of polymerization of $P(I-n)(n=1-9)$ as calculated by GPC relative to polystyrene standards varied from 35 to 72 , with polydispersities of 1.08-1.16, demonstrating that ROMP can be used to polymerize monomers containing phenylbenzoate groups in a controlled manner. Although the $\mathrm{P}(\mathrm{I}-\mathrm{n})$ polymers do form fluid mesophases, their glass transitions are difficult to detect and they decompose before undergoing isotropization. The fluid nature of the mesophase is masked by simultaneous decomposition and apparently oxidative crosslinking at elevated temperatures. The poly(II-n) series were therefore prepared to determine the effect of molecular weight on the thermotropic behavior of SCLCPs with laterally attached mesogens. The transition temperatures of poly(II-n) are lower than those of poly $(I-n)$ due to the use of a short spacer connecting the polymer and mesogen, and due to the polymers' regioirregularity. Since the mesogen is asymmetric, head-to-head, head-to-tail, and tail-to-tail sequences of endo- and exo- substituted repeat units are possible, as well as the presence of both cis and trans double bonds. They exhibit enantiotropic nematic mesophases in addition to the glass transition. As shown in Figure 2, both the glass and nematic-isotropic transition temperatures of poly(II-1) become independent of molecular weight at approximately 25 repeat units, which evidently corresponds to the persistence length of these laterally attached SCLCPs. Both the glass transition and isotropization temperatures of poly(II-n) decrease as the n-alkoxy length increases. Isotropization decreases with an oddeven alternation, with the even-membered substituents having broader and more stable mesophases than the odd-membered substituents.

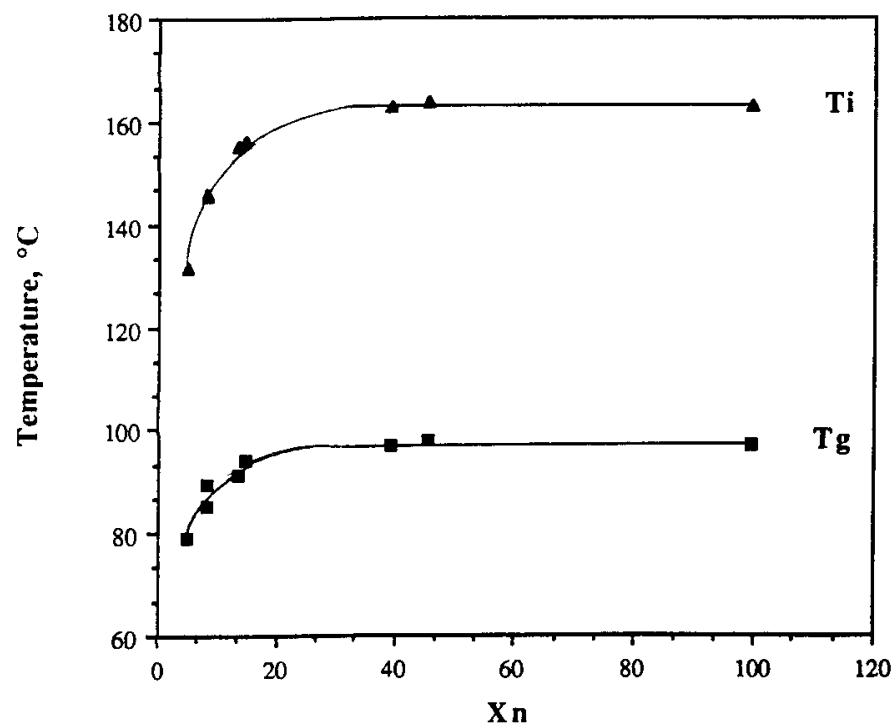

Figure 2. Dependence of the glass and nematic-isotropic transitions of poly(II-1) on the GPC determined degree of polvmerization. 


\section{ADVANTAGES AND DISADVANTAGES SCLCPS PREPARED BY ROMP}

Using a combination of high vacuum line and drybox techniques, mesogenic norbornene derivatives with non-protonic functional groups can be polymerized by ROMP to obtain polymers with controlled molecular weight and narrow molecular weight distributions. Polymers with degrees of polymerization from a few repeat units to several hundred are routinely prepared. However, every repeat unit of the resulting polymer backbone contains a double bond and two allylic hydrogens. The double bonds could be used advantageously as a site for further functionalization and/or crosslinking, such as for preparing chiral liquid crystalline elastomers. Nevertheless, the polymers are easily oxidized due to this high concentration of double bonds and allylic hydrogens (33). Allylic groups react photo-

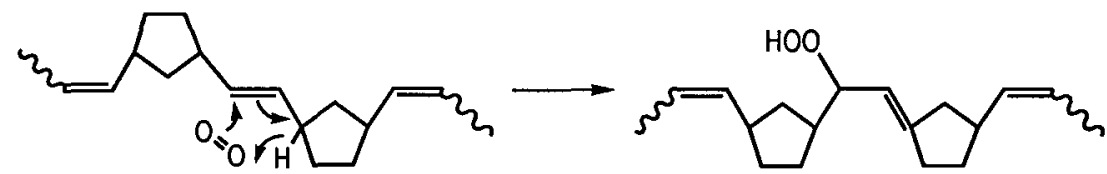

chemically with singlet oxygen in an ene type reaction, or free radically with triplet oxygen, to produce hydroperoxides. The hydroperoxides then induce free radical crosslinking.

\section{ACKNOWLEDGMENT}

Acknowledgment is made to the donors of The Petroleum Research Fund, administered by the ACS, for financial support.

\section{REFERENCES}

(1) (a) V. Percec and C. Pugh, in Side Chain Liquid Crystalline Polymers, ed. C.B. McArdle, Chapman and Hall, New York, 1989, ch. 3; (b) V. Percec and D. Tomazos, in Comprehensive Polymer Science, First Supplement, eds. S.L. Aggarwal and S. Russo, Pergamon Press, Oxford, 1992, ch. 14.

(2) See for example: (a) V. Percec, D. Tomazos and C. Pugh, Macromolecules, 22, 3259 (1989); (b) T. Sagane and R. Lenz, Macromolecules, 22, 3763 (1989); (c) V. Percec and M. Lee, Macromolecules, 24, 2780 (1991); (d) V. Percec and D. Tomazos, Adv Mater., 4, 548 (1992).

(3) A.J. Amass, in Comprehensive Polymer Science, Vol. 4, eds. G. Allen and J.C. Bevington, Pergamon Press, Oxford, 1989, ch. 6; and references therein.

(4) W. Feast, in Comprehensive Polymer Science, Vol. 4, eds. G. Allen and J.C. Bevington, Pergamon Press, Oxford, 1989, ch. 7; and references therein.

(5) L.R. Gilliom and R.H. Grubbs, J. Am. Chem. Soc., 108, 733 (1986).

(6) K.C. Wallace and R.R. Schrock, Macromolecules, 20, 448 (1987).

(7) J. Kress, J.A. Osborn, R.M.E. Greene, K.J. Ivin and J.J. Rooney, L.Am. Chem. Soc, 109,899 (1987).

(8) G.C. Bazan, J.H. Oskam, H.-N. Cho, L.Y. Park and R.R. Schrock, J.Am. Chem. Soc, $113,6899(1991)$.

(9) L.F. Cannizzo and R.H. Grubbs. Macromolecules. 20. 1488 (1987). 
(10) (a) W.J. Feast, V.C. Gibson E. Khosravi, E.L. Marshall and J.P. Mitchell, Polymer, 33, 872 (1992); (b) W.J. Feast, V.C. Gibson and E.L. Marshall, J. Chem. Soc. Chem. Commun, 1157 (1992).

(11) M. Szwarc, Carbanions, Living Polymers and Electron Transfer Processes, Interscience Publishers, New York (1968).

(12) S. Penczek, P. Kubisa and R. Szymanski, Makromol. Chem., Rapid Commun., 12, 77 (1991).

(13) (a) K. Matyjaszewski, J. Polym. Sci. Polym. Chem. Ed, 31, 995 (1993); (b) K. Matyjaszewski, Macromolecules, 26, 1787 (1993).

(14) R.R. Schrock, Acc. Chem. Res. 24, 158 (1990); and references therein.

(15) R.R. Schrock, J. Feldman, L.F. Canizzo and R.H. Grubbs, Macromolecules, 20, 1169 (1987).

(16) (a) R.R. Schrock, R. DePue, J. Feldman, C.J. Schaverien, J.C. Dewan and A. Liu, J. Am. Chem. Soc., 110, 1423 (1988); (b) R.T. DePue, R.R. Schrock, J. Feldman, K. Yap, D.C. Yang, W.M. Davis, L. Park, M. DiMare, M. Schofield, J. Anhaus, E. Walborsky, E. Evitt, C. Kruger and P. Betz, Organometallics, 9, 2262 (1990).

(17) R.R. Schrock, J.S. Murdzek, G.C. Bazan, J. Robbins, M. DiMare and M. O'Regan, L Am. Chem. Soc., 112, 3875 (1990).

(18) H.H. Fox, K.B. Yap, J. Robbins, S. Cai and R.R. Schrock, Inorg, Chem., 31, 2287 (1992).

(19) (a) J.S. Murdzek and R.R. Schrock, Macromolecules, 20, 2640 (1987); (b) G. Bazan, R.R. Schrock, E. Khosravi, W.J. Feast and V.C. Gibson, Polymer Commun, 30, 258 (1989); (c) G.C. Bazan, E. Khosravi, R.R. Schrock, W.J. Feast, V.C. Gibson, M.B. O'Regan, J.K. Thomas and W.M. Davis, L.Am. Chem. Soc, 112, 8378 (1990); (d) G.C. Bazan, R.R. Schrock, H.-N. Cho and V.C. Gibson, Macromolecules, 24, 4495 (1991).

(20) (a) V. Sankaran, C.C. Cummins, R.R. Schrock, R.E. Cohen and R.J. Silbey, J. Am. Chem. Soc., 112, 6858 (1990); (b) C.C. Cummins, M.D. Beachy, R.R. Schrock, M.G. Vale, V. Sankaran and R.E. Cohen, Chem. Mater, 3, 1153 (1991) (c) Y.N.C. Chan, R.R. Schrock and R.E. Cohen, Chem. Mater, 4, 24 (1992); (d) C.C. Cummins, R.R. Schrock and R.E. Cohen, Chem. Mater, 4, 27 (1992).

(21) J.P. Mitchell, V.C. Gibson and R.R. Schrock, Macromolecules, 24, 1220 (1991).

(22) L. Gold, L.Chem. Phys, 28, 91 (1958).

(23) G.C. Bazan and R.R. Schrock, Macromolecules, 24, 817 (1990).

(24) Z.Wu, D.R. Wheeler and R.H. Grubbs, J.Am. Chem. Soc, 114, 146 (1992).

(25) Z. Komiya, C. Pugh and R.R. Schrock, Macromolecules, 25, 3609 (1992).

(26) Z. Komiya, C. Pugh and R.R. Schrock, Macromolecules, 25, 6586 (1992).

(27) Z. Komiya and R.R. Schrock, Macromolecules, 26, 1393 (1993).

(28) S.-H. Kim, H.-J. Lee, S.-Ho Jin, H.-N. Cho and S.-K. Choi, Macromolecules, 26, 846 (1993).

(29) (a) H.F. Leube and H. Finkelmann, Makromol. Chem. 191, 1317 (1991); (b) H.F. 
Leube and H. Finkelmann, ibid, 192, 1317 (1991).

(30) F. Hardouin, S. Mery, M.F. Achard, L. Noirez and P. Keller, L. Phys. II, 1511 (1991).

(31) C. Pugh and R.R. Schrock, Macromolecules, 25, 6593 (1992).

(32) (a) Q.-F. Zhou, H.-M. Li and X.-D. Feng, Macromolecules, 20, 233 (1987); (b) Q.-F. Zhou, H.-M. Li and X.-D. Feng, Mol. Cryst. Liq. Cryst., 155, 73 (1988).

(33) I.F.A.F. El-Saafin and W.J. Feast, L.Mol. Catal, 15, 61 (1982). 